\title{
INFORMATION TECHNOLOGY OF LASER COAgUlation STRATEgy SELECTION IN DIABETIC RETINOPATHY TREATMENT
}

\author{
Shirokanev Alexander ${ }^{1,2}$, Kibitkina Alena ${ }^{2}$, Ilyasova Nataly ${ }^{1,2}$ and \\ Zamyckij Evgeny ${ }^{3}$ \\ ${ }^{1}$ IPSI RAS - branch of the FSRC «Crystallography and Photonics» RAS, \\ Molodogvardejskaya street 151, Samara, Russia, 443001 \\ ${ }^{2}$ Samara National Research University, Moskovskoe Shosse 34A, Samara, \\ Russia, 443086 \\ ${ }^{3}$ Samara State Medical University, Chapayevskaya street 89, Samara, Russia, \\ 443099
}

\begin{abstract}
Diabetic retinopathy is frequent, the most dangerous fundus disease. Diabetic retinopathy can result in many serious diseases. For various reasons, patients lose vision in untimely or incorrect treatment of diabetic retinopathy. The current method of treating diabetic retinopathy is laser coagulation. The ophthalmologist decides which zones need to be shelled to reduce edema based on his experience. Laser radiation parameters and distance between laser shots are also selected based on the experience of previous operations. However, the accuracy of the selection of these parameters can affect the result of treatment. Achieving high accuracy is empirical difficult. The present paper proposes a technology for selecting an effective laser coagulation strategy consisting in application of a genetic efficiency optimization algorithm based on solving of the problems of mathematical simulation of laser burns. Technology solves the problem of choosing accurate laser coagulation parameters.
\end{abstract}

\section{KEYWORDS}

Diabetic retinopathy, laser coagulation, ocular fundus, information technology, mathematical modeling, thermal conductivity equation

\section{INTRODUCTION}

One of the most dangerous and common endocrine diseases is diabetes mellitus, retinal blood vessels are affected, resulting in diabetic retinopathy (DR). The DR condition affects all parts of the retina, but changes in its central part, which are diabetic macular edema, lead to very rapid and irreversible loss of vision [1]. Vision loss can be prevented in more than $50 \%$ of cases if accurate diagnosis was performed in a timely manner and treatment was effective [2-4]. Worldwide, laser photocoagulation is a standard treatment. Its effectiveness was confirmed during a major study (ETDRS, 1987) [5].

Laser coagulation is the application of a series of dosed microburn in the macular edema region. Laser coagulation causes coagulations form to prevent hemorrhages. The effectiveness of the treatment result is influenced by factors such as the energy of laser radiation converted to heat, the distance between coagulates, and the area of the coating. The presented parameters are 
currently selected based on the doctor experience of previous operations. However, the distance between shots and the energy of thermal radiation can greatly affect the achievement of high treatment efficiency. Based on the thermal conductivity equation, heat propagation in the retina can be simulated. The distance the heat spreads determines the therapeutic effect in the local area. Pathological elements such as exudates or edema are usually determined by retinal thickness. After coagulation formation, the retina is gradually brought to normal. However, simulating laser radiation on the retina at temperature dependent coefficients result in a strong nonlinearity of the problem. Nevertheless, heat propagation can be assessed on a static thermal conductivity task as well.

The effectiveness of the diabetic retinopathy treatment strategy can be assessed by analyzing the magnitude of critical area and regions with therapeutic effect [6]. By critical area will be understood an area whose temperature is higher than critical. This temperature damages the retina. The therapeutic effect is determined by pathological regions in which the temperature is not critical but causes the formation of a coagulate, i.e. reaches the pigment epithelium - a narrow boundary layer between the retina and the sclera.

\section{Laser Coagulation Simulation Based on Thermal CONDUCTIVITY EQUATION}

Laser radiation contributes to the conversion of electromagnetic field energy into thermal energy $[7,8]$. Mathematically, we can derive the initial condition for a thermal conductivity equation through a solution to Maxwell 's equation. Given the simplicity of the problem, it is not necessary to solve Maxwell 's equation. Initial condition deduced through intensity of laser radiation.

Laser coagulation modeling can be implemented using the equation (1). A problem has an initial condition defined by the formula (2), and first boundary conditions.

$$
\begin{gathered}
c_{o \sigma}(x, y, z) \frac{\partial T}{\partial t}=\beta(x, y, z)\left(\frac{\partial^{2} T}{\partial x^{2}}+\frac{\partial^{2} T}{\partial y^{2}}+\frac{\partial^{2} T}{\partial z^{2}}\right) . \\
T_{t=0}^{x_{0} y_{0}}=\psi_{x_{0} y_{0}}(x, y, z)=\frac{I\left(\sqrt{\left(x-x_{0}\right)^{2}+\left(y-y_{0}\right)^{2}}\right) \beta(x, y, z) e^{-\beta(x, y, z) z} \Delta t}{c_{o \sigma}(x, y, z)}+T_{c}(x, y, z),
\end{gathered}
$$

where $T_{c}$ - total temperature obtained from previous microburn;

$\beta$ - absorption coefficient;

$c_{o \sigma}$ - thermal capacity;

$I(r)$ - intensity of laser radiation.

Consider the section of the three-dimensional space of the layers. Get a two-dimensional problem (5), $n$ which $z$ defines the depth of the layers and $\mathrm{x}$ defines the offset along the section.

$$
c_{o \sigma}(x, z) \frac{\partial T}{\partial t}=\beta(x, z)\left(\frac{\partial^{2} T}{\partial x^{2}}+\frac{\partial^{2} T}{\partial z^{2}}\right)
$$

Instead of a three-dimensional problem, we can consider two-dimensional problems for every two shots in pairs. 
The criterion for coagulum formation can be considered heating the pigment epithelium to the desired temperature. Achieving the heat of the pigment epithelium depends on the shape of the retina at a particular point. Figure 1a shows the temperature distributions at the center of the shot at $1 \mathrm{~ms}$. After this time, cooling occurs. Figure $1 \mathrm{~b}$ shows the temperature distributions in retinal.

Adjacent shots can result in a critical temperature in the crossing area. Coagulants must be located sufficiently apart to unduly damage the retina.

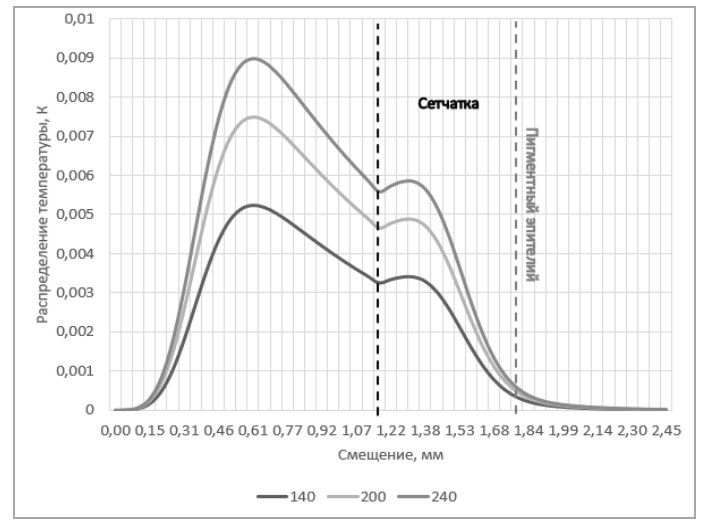

(a)

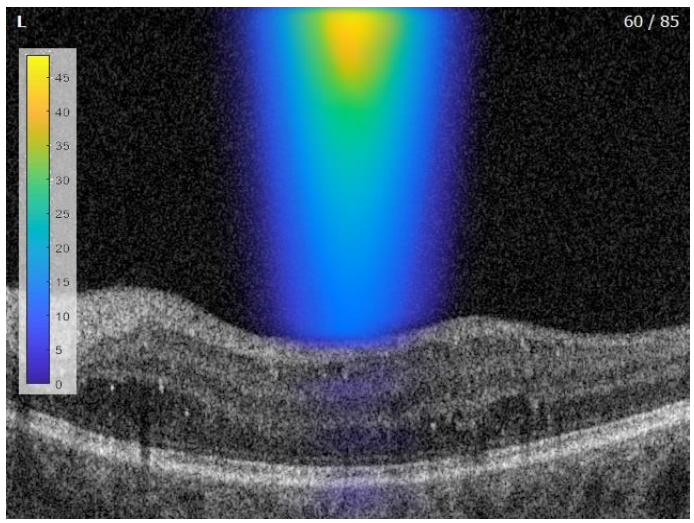

(б)

Figure 1. Heat propagation distance: (a) in the one-dimension case by different laser powers; (б) in the two-dimensional case

\section{Information Technology fOR Selecting EFfective LASER COAgulation STRATEgY In Diabetic RETINOPATHY TrEatMENT}

The technology is based on the application of a genetic algorithm to find an effective laser coagulation strategy. In order to describe the work of the genetic algorithm, it is necessary to introduce the adaptation function of the individual. Adaptation will be determined by the effectiveness of the coagulation plan.

To generate an initial population, we will use the algorithm of formation of coagulation plan "Random map," presented in the work [9]. The main diagram of the technology is shown in figure 2.

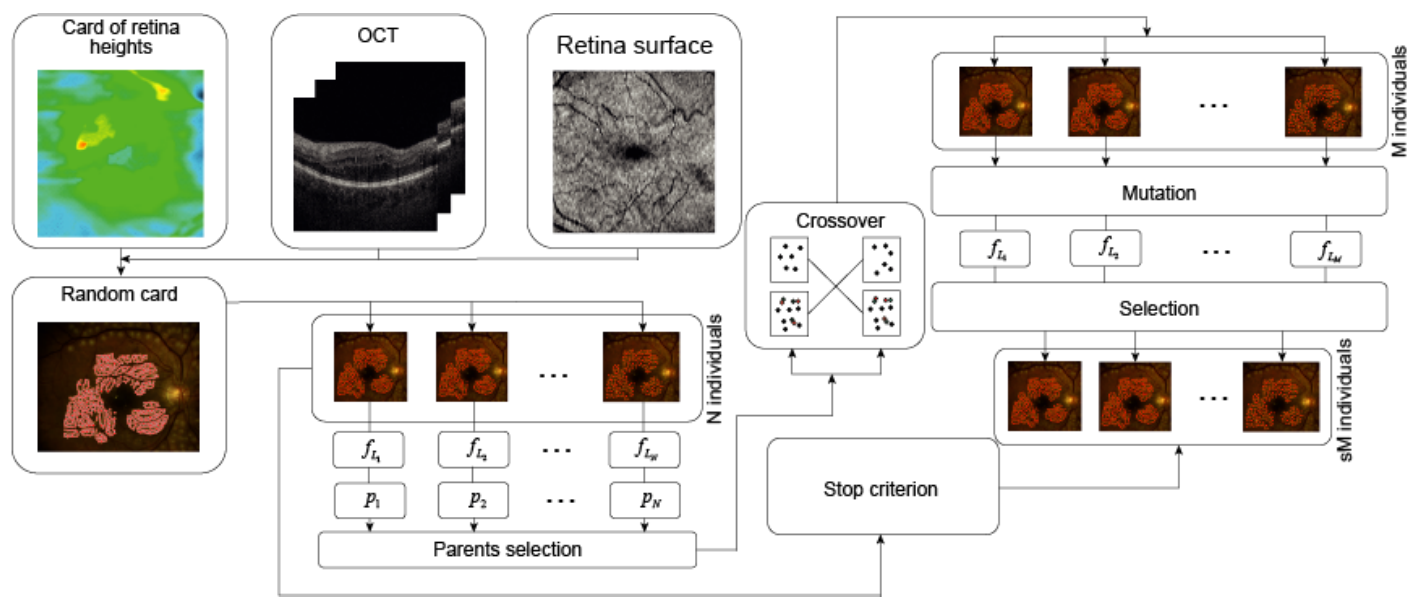


Figure 2. Scheme of Technology for Selecting an Effective Laser Coagulation Strategy in the Treatment of Diabetic Retinopathy

The stages of crossing and mutation will be based on the phenotypic form of similar parents $[10,11]$ and the random distribution of nodes using a normal distribution with a sufficiently small dispersion. The selection will be carried out according to the roulette principle $[10,11]$.

The main difficulty of the technology is the need for modeling to calculate the adaptation function of a given individual. Thus, calculating the adaptation function is the most computationally complex task. This greatly slows down the execution of the genetic algorithm. On average, solving a two-dimensional thermal conductivity problem for two shots on a sufficiently small modeling area using a primitive sequential algorithm can be greater than 1 minute. It may take more than an hour to calculate the fitting function. This means that a solution on the middle area can be formed for several months. This requires high-performance simulation using cluster systems and graphics accelerators. CUDA technology is best suited to this task because the main operations to be accelerated are matrix calculations that are easily vectorized.

\section{Conclusions}

The present paper proposes a technology for selecting an effective laser coagulation strategy for the treatment of diabetic retinopathy based on the use of a genetic algorithm. The technology has an excessively high computational complexity, which requires high-performance mathematical modeling algorithms and additional solution simplification. Numerical modeling is recommended using CUDA technology.

With this technology, the coordinates of the nodes can be optimized quite accurately. However, power is not involved in the stages of the genetic algorithm, so optimization of node capacity is extremely difficult. Mainly node coordinates are optimized. However, individuals who are a plan of coagulants with misused capacities are not selected. Therefore, the capacity will not be absolutely random, but will be with a marked error. However, this is sufficient to meet the challenge.

\section{ACKNOWLEDGEMENTS}

This work was financially supported by the Russian Foundation for Basic Research under grant \# 19-29-01135, \# 17-01-00972, \# 19-31-90160 and by the Ministry of Science and Higher Education within the State assignment to the FSRC "Crystallography and Photonics" RAS.

\section{REFERENCES}

[1] Doga, A. \& Kachalina, G. \& Pedanova, E. \& Buryakov, D, (2014) "Modern diagnostic and treatment aspects of diabetic macular edema", Ophthalmology, Diabetes, Vol. 4, pp. 51-59.

[2] Zamytskiy, E, (2017) "Analysis of the coagulates intensity in laser treatment of diabetic macular edema in a Navilas robotic laser system", Saratov Journal of Medical Scientific Research, Vol. 13(2), pp. 375-378.

[3] Ilyasova, N, (2014) "Evaluation of geometric features of the spatial structure of blood vessels", Computer Optics, Vol. 38(3), pp. 529-538.

[4] Khorin, P. \& Ilyasova, N. \& Paringer, R. (2018) "Informative feature selection based on the Zernike polynomial coefficients for various pathologies of the human eye cornea", Computer Optics, Vol. 42(1), pp. 159-166. 
[5] Astakhov, Y. \& Shadrichev, F. \& Krasavina, M. \& Grigoryeva, N, (2009) "Modern approaches to the treatment of a diabetic macular edema", Ophthalmologic sheet, Vol. 4, pp. 59-69.

[6] Kozak, I. \& Jeffrey Luttrull, (2014) "Modern retinal laser therapy", Saudi Journal of Ophthalmology, Vol. 29(2), pp.137-146.

[7] Fasano, A. \& Hömberg, D. \& Naumov D, (2010) “On a mathematical model for laser-induced thermotherapy”, Applied Mathematical Modelling, Vol. 34, pp. 3831-3840.

[8] Jiang, S. C. \& Zhang, X. X, (2006) "Mathematical modeling of dynamic laser coagulation during laser-induced interstitial thermotherapy", Fourth International Conference on Photonics and Imaging in Biology and Medicine, Proceedings Volume 6047.

[9] Shirokanev, A. \& Kirsh, D. \& Ilyasova, N. \& Kupriyanov, A, (2018) "Investigation of algorithms for coagulate arrangement in fundus images”, Computer Optics, Vol. 42(4), pp. 712-721.

[10] Ghaheri, A. \& Shoar, S. \& Naderan, M. \& Shahabuddin, S, (2015) "The Applications of Genetic Algorithms in Medicine", Oman Medical Journal, Vol. 30(6), pp.406-416.

[11] Artemov, S. \& Belyaev, A. \& Bushukina, O. \& Khrushchalina, S. \& Kostin, S. \& Lyapin, A. \& Ryabochkina, P. \& Taratynova, A, (2019) "Endovenous laser coagulation using two-micron laser radiation: mathematical modeling and in vivo experiments", Proceedings of «International Conference on Advanced Laser Technologies (ALT)», Vol. 19. P. 1. 


\title{
AuThORS
}

\section{Alexander Shirokanev}

Shirokanev A S (b. 1993), graduated (2017) with a master's degree in Applied Mathematics and Informatics. At present, he is a Junior Researcher at the Image Processing Systems Institute of RAS - Branch of the FSRC "Crystallography and Photonics" RAS, and holding a part-time position of an assistant at SSAU's Technical Cybernetics sub-department. At present he is an assistant of the Technical Cybernetics department at Samara University. The area of interests includes digital image processing, mathematical modeling, numerical analysis and intellectual analysis of medical images. E-mail: alexandrshirokanev@gmail.com.

\begin{abstract}
Alena Kibitkina
Kibitkina A S(b. 1997), graduated (2014) from Samara school. At present, she is a student at Samara University Technical Cybernetics sub-department. E-mail: anacy1222@gmail.com.
\end{abstract}

\section{Ilyasova Nataly}

Ilyasova N Yu (b. 1966), graduated with honors from S.P. Korolyov Samara State Aerospace University (SSAU) (1991). She received her PhD (1997) and DSc (2015) in Technical Sciences. At present, she is a Senior Researcher at the Image Processing Systems Institute of RAS - Branch of the FSRC "Crystallography and Photonics" RAS, and holding a part-time position of Professor at SSAU's Technical Cybernetics sub-department. The area of interests includes digital signals and image processing, pattern recognition and artificial intelligence, biomedical imaging and analysis. She's list of publications contains more than 130 scientific papers, including 45 articles and 3 monographs published with coauthors. E-mail: ilyasova@smr.ru.

\section{Zamytskiy Evgeniy}

Zamytskiy E A - postgraduate Ophthalmology Department Samara State Medical University and ophthalmologist Ophthalmoendocrinology Department Samara Regional Clinical Ophthalmological Hospital named after T.I. Eroshevsky. Research interests: diabetic retinopathy, diabetic macular edema, diabetic macular ede-ma laser treatment.
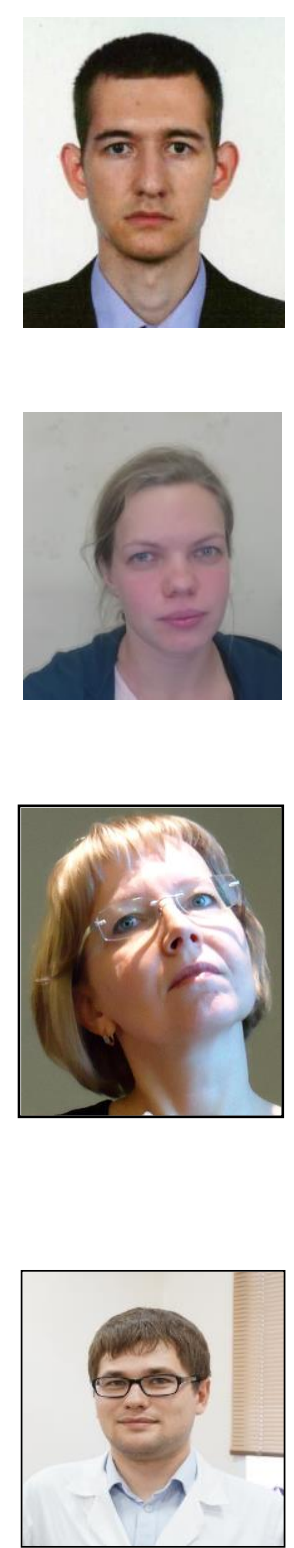